\title{
LATERAL HYPOTHALAMIC MODULATION OF ORAL SENSORY AFFERENT ACTIVITY IN NUCLEUS TRACTUS SOLITARIUS NEURONS OF RATS ${ }^{1}$
}

\author{
RYUJI MATSUO, ${ }^{2}$ NOBUAKI SHIMIZU, ${ }^{3}$ AND KIYOSHI KUSANO ${ }^{4}$ \\ Neurophysiology Laboratory, Department of Biology, Illinois Institute of Technology, Chicago, Illinois 60616
}

Received June 27, 1983; Revised November 29, 1983; Accepted January 3, 1984

\begin{abstract}
This study was designed to examine whether the sensory afferents from the anterior part of the tongue are modulated by activity of the lateral hypothalamic area (LHA) at the level of the nucleus tractus solitarius (NTS) in rats. The electrical activity of the NTS neurons was recorded extracellularly, and they were classified as gustatory, thermal, or mechanical neurons in accordance with their responsiveness to tongue stimulation by taste solutions, by warm and cool water, and by stroking the tongue surface. Sixty-two percent of the neurons were polysynaptically activated by electrical stimulation of the LHA. When a single conditioning stimulus of the LHA was applied prior to the test electrical stimulation of the tongue at various conditioning-test intervals, the activity of the gustatory neurons was facilitated by 30 to $80 \%$ of their control level for a period of approximately 20 to $150 \mathrm{msec}$. The activity of the mechanical and thermal neurons was suppressed for a 50 - to $300-\mathrm{msec}$ period. These results demonstrate the existence of modulatory effects from the LHA on the NTS neurons, which receive sensory information from the tongue.
\end{abstract}

A number of studies have indicated that electrical and chemical stimulation of the lateral hypothalamic area (LHA) can elicit eating, drinking, gnawing, and exploratory behaviors. Although some of these behaviors (or fractional components of them) can also be evoked by stimulation of the cortex, amygdala, thalamus, and lower brainstem, the LHA is the most effective region in terms of the integrated feeding behavior (see Powley, 1977; Le Magnen, 1983). In this sense, the LHA is known to be a locus which receives information from the internal environment (postingestional signals of humoral origin) as well as from external neural inputs associated with, and elicited by, feeding. As to the external signals, olfactory, visual, trigeminal, and gustatory projections to the LHA have been demonstrated (Norgren, 1970; Scott and Pfaffmann, 1972; Rolls et al., 1976; Riley et al., 1981). Under the influence of both humoral and neural factors, it has been suggested that the LHA not only controls the motor centers in the medulla related to ingestive behavior but also the sensory afferent inputs, because a sensory

\footnotetext{
${ }^{1}$ This work was supported in part by National Institutes of Health Grant NS 12275.

${ }^{2}$ Present address: Department of Oral Physiology, Osaka University Faculty of Dentistry, 1-8 Yamadaoka, Suita, Osaka 565, Japan.

${ }^{3}$ Present address: Department of Physiology, Faculty of Medicine, Kyushu University, Fukuoka 812, Japan.

${ }^{4}$ To whom correspondence should be addressed.
}

neglect to olfactory, visual, and somatosensory stimuli has been produced following LHA lesions (Marshall et al., 1971). Moreover, recent neuroanatomical studies (Saper et al., 1976; Roberts, 1980; Hosoya and Matsushita, 1981) have revealed descending projections from the LHA to the nucleus tractus solitarius (NTS) and the parabrachial nucleus, which contain the second- and third-order gustatory neurons, respectively. Additionally, from electrophysiological studies, Bereiter et al. (1980) reported that the brainstem neurons responsive to oropharyngeal stimulation received hypothalamic inputs. However, it is not yet clear how the LHA activity modified the afferent inputs from the tongue at the level of the NTS neurons.

In this experiment, the neural activity of the NTS neurons responsive to mechanical, thermal, and/or gustatory stimulation applied to the anterior part of the tongue of rats was recorded; additionally, the effect of the LHA stimulation on the NTS activity was analyzed.

\section{Materials and Methods}

Twenty male Sprague-Dawley rats weighing 300 to 390 gm were used. The animals were anesthetized by intraperitoneal injection of a mixture of $\alpha$-chloralose (50 mg/ $\mathrm{kg}$ ) and urethane $(500 \mathrm{mg} / \mathrm{kg})$. Whenever the flexion withdrawal reflex evoked by pinching the skin of the foot appeared, maintenance doses of urethane were supplied 
throughout the experiment via a cannula placed in the femoral vein. After a tracheal cannulation, the animal was placed in a stereotaxic instrument using nontraumatic ear bars to avoid damaging the chorda tympani nerve.

Stimulating the lateral hypothalamic area. The dorsal surface of the skull was exposed and aligned horizontally. The coordinates used for the LHA were 2.0 to $2.2 \mathrm{~mm}$ posterior to the bregma, 1.3 to $1.5 \mathrm{~mm}$ lateral to the midline (sagittal suture), and 7.5 to $8.0 \mathrm{~mm}$ below the surface of the brain. The electrode was inserted with a micromanipulator through a small hole drilled in the skull, and it was then anchored firmly to the skull by screws and dental acrylic resin. In this way the tip of the electrode was always positioned in the LHA region medial to the tractus opticus and ventrolateral to the fornix at the level of the ventromedial nucleus. Stimulating electrodes were commercially prepared stainless steel coaxial electrodes with a tip diameter of $100 \mu \mathrm{m}$ (Rhodes Medical Instruments, Model SNE-100). Electrical stimuli were square wave pulses of 0.1 - to 0.3 -msec duration and $0.1-$ to $0.2-\mathrm{mA}$ intensity at $0.5-$ to $100-\mathrm{Hz}$ frequency, with the tip of the electrode cathodal. They were supplied by a Grass S48 stimulator with an isolation unit.

Recording the neural activity in the NTS. After implanting the LHA stimulating electrode, the posterior portion of the skull was removed and the posterior cerebellum was aspirated to expose the surface of the brainstem from the obex to the dorsal cochlear nuclei. The surface of the exposed medulla was covered with a warm mineral oil. Pneumothorax was performed in some experiments, and respiration was maintained artificially. Extracellular neuronal activity was recorded using either glass micropipettes (tip diameter of 1.0 to $4.0 \mu \mathrm{m}$ ) filled with $2 \mathrm{M} \mathrm{NaCl}$ or commercially made stainless steel microelectrodes with impedances of about 10 megohms. With the obex as a reference point, the electrode was positioned and advanced into the NTS by using a microdrive electrode holder. A small chloridized $\mathrm{Ag} / \mathrm{AgCl}$ plate was placed on surrounding tissues as an indifferent electrode. The potentials were led to a Grass P15 a-c preamplifier and then to a Tektronix 5113 oscilloscope and an audiomonitoring circuit. The amplified potentials were stored by a Vetter Instrumentation recorder model $B$ and later photographed.

Responsiveness of the recorded NTS neurons was tested by applying taste, tactile, thermal, and electrical stimulations to the tongue. Taste stimuli made up with distilled water consisted of $0.5 \mathrm{M}$ and $1.0 \mathrm{M}$ sucrose, 0.1 $\mathrm{M}$ and $0.5 \mathrm{M} \mathrm{NaCl}, 0.01 \mathrm{M}$ and $0.03 \mathrm{M} \mathrm{HCl}$, and $0.01 \mathrm{M}$ and $0.02 \mathrm{M}$ quinine- $\mathrm{HCl}$. About $3 \mathrm{ml}$ of a test solution were poured by gravity flow $(10 \mathrm{ml} / \mathrm{sec})$ to the tongue through a burette which was held just above the center of the dorsal tongue surface. With this method the anterior one-half of the tongue was stimulated. Tap water was applied to the tongue for rinsing after $15 \mathrm{sec}$ of stimulus presentation time. Rinsing was usually continued for at least $30 \mathrm{sec}$. Taste solutions and rinsing water were at room temperature (about $24^{\circ} \mathrm{C}$ ). Mechanical stimulation was applied by a small glass rod. Cooling and warming the tongue were performed by applying cold $\left(5^{\circ} \mathrm{C}\right)$ and warm $\left(40^{\circ} \mathrm{C}\right)$ deionized water to the tongue surface. Electrical stimulation of the tongue was delivered through a pair of stainless steel wire electrodes (200 $\mu \mathrm{m}$ in diameter, $3 \mathrm{~mm}$ interpolar distance) insulated except for $1 \mathrm{~mm}$ of the tip. The electrodes were inserted from the ventral side of the tongue into the subcutaneous tissues of the anterior and dorsal part of the tongue. Electrical stimuli were square wave pulses of $0.3-\mathrm{msec}$ duration and 2.0 - to $6.0-\mathrm{mA}$ intensity at $0.5-$ to $100-\mathrm{Hz}$ frequency.

At the end of the experiment the brain tissue was electrically coagulated by passing currents through the stimulating electrode $(20 \mu \mathrm{A}, 10 \mathrm{sec})$ and the recording metal electrode ( $15 \mu \mathrm{A}, 15 \mathrm{sec})$; the locations of the tips of the electrodes were later verified histologically.

\section{Results}

A total of 37 neuron discharges were sampled from the most rostral NTS area previously reported to receive primary sensory afferents from the ipsilateral anterior part of the tongue in rats (Blomquist and Antem, 1965; Halpern and Nelson, 1965). When the electrical stimulation was applied to the ipsilateral anterior part of the tongue, these neurons showed the following features, which were characteristic of postsynaptic responses to synchronous afferent volleys. At just above threshold intensity, the spike latencies were relatively longer ( 1 to $2 \mathrm{msec}$ ) and more variable (within 1 to $2 \mathrm{msec}$ ) as compared to the situation where higher stimulus intensities were employed. At over $30-$ to $50-\mathrm{Hz}$ repetition rate, the spike discharges did not always occur on stimulation. In addition, the spike latencies increased a few milliseconds and became more unstable.These electrophysiological features corresponded to the criteria which were employed by Doetsch and Erickson (1970) to discriminate the neural activity arising from postsynaptic cells in the NTS from that of first-order fibers projecting to the NTS. Out of these 37 neuron spikes, $23(62 \%)$ were also responsive to the electrical stimulation applied to the ipsilateral LHA at the level of the ventromedial nucleus; the remaining $14(38 \%)$ neurons were not responsive.

As shown in the recordings in Figure 1, there were no remarkable differences in frequency-following capability to tongue stimulation, whereas the pattern of activation produced by the LIIA stimulation differed from one neuron to another. For example, the spikes in Figure $1 A$ followed the stimulation at a frequency of $10 \mathrm{~Hz}$, with a latency of $10 \mathrm{msec}$ but sometimes failed to respond to each stimulus at $20 \mathrm{~Hz}$. The neuron in Figure $1 B$ produced multiple spike discharges with an unstable longer latency but did not follow with spike discharges at over $25-\mathrm{Hz}$ stimulus frequency. These findings suggest that these NTS neurons are polysynaptically activated by LHA stimulation. Slow potentials lasting from 3 to 40 msec were recorded in all cases when the LHA stimulation was applied at low frequencies (less than $10 \mathrm{~Hz}$ ). Since these potentials greatly attenuated and disappeared at over $30-\mathrm{Hz}$ repetition rate (Fig. $1 B$ ) and since the spikes occurred within a range of the time course of the waves, they seemed to be originating from postsynaptic potentials. In Figure $1 C$, such slow potentials were provoked by the LHA stimulation, whereas spike dis- 


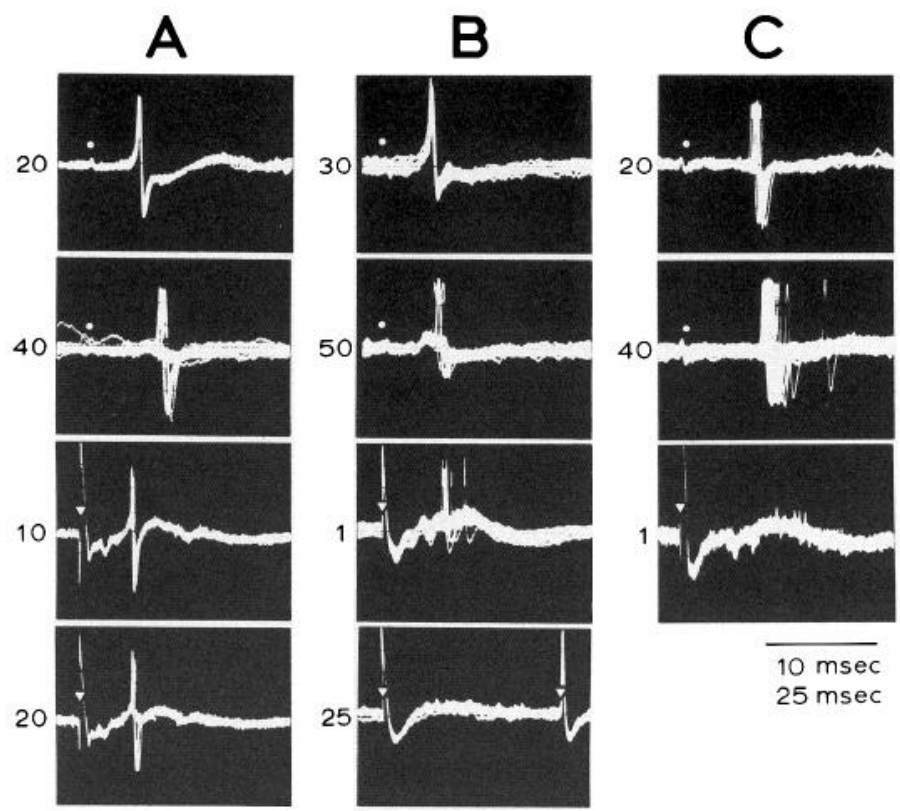

Figure 1. Examples of spike discharges recorded from neurons in the NTS. Each recording shows over 10 superimposed sweeps. Circles and triangles indicate electrical stimulations of the tongue and the LHA, respectively. Stimulus repetition rates are at the side of each tracing. Column A, Tongue and LHAresponsive neuron. The upper two traces were obtained by ipsilateral tongue stimulation. At a frequency of $20 \mathrm{~Hz}$, constant latency of spike discharges was observed. At $40 \mathrm{~Hz}$, the neuron failed to respond to each stimulus; a small latency variation was observed. The lower two traces show the responsiveness to the LHA stimulation. Spike discharges in response to each stimulus at a frequency of $10 \mathrm{~Hz}$ were superimposed on the evoked potential lasting from 10 to $50 \mathrm{msec}$. At $20 \mathrm{~Hz}$, a failure to respond to each stimulus was noticed along with a decrease in the amplitude of the evoked slow potential. Column $B$, Tongue and LHA-responsive neuron. The effects of increasing frequency of tongue stimulation were similar to those illustrated in column $A$; in contrast, multiple spike discharges occurred with an unstable latency in response to the LHA stimulation at $1 \mathrm{~Hz}$. At $25 \mathrm{~Hz}$, spike discharges did not follow the stimulus frequency and the evoked potential was markedly eliminated. (The record does not show the initial stimulus, and, therefore, spike discharges are not shown.) Column C, LHAnonresponsive neuron. The frequency-following capability to tongue stimulation was essentially the same as that of the neurons in columns $A$ and $B$. By the LHA stimulation (third record), a slow evoked potential lasting 10 to $50 \mathrm{msec}$ was evoked, but spike discharges were not produced even if strong stimulation (up to $0.2 \mathrm{~mA}, 0.3 \mathrm{msec}$ ) was applied. Time calibration, $10 \mathrm{msec}$ for responses to tongue stimulation and $25 \mathrm{msec}$ for responses to the LHA stimulation.

charges were not driven even if strong stimuli (up to 0.2$\mathrm{mA}$ intensity and $0.3-\mathrm{msec}$ duration) were applied. This intensity was approximately 2 times higher than the intensity which produced the spike discharges in the LHA-responsive neurons. We did not employ much stronger stimulation (over $0.4 \mathrm{~mA}$ or more than $0.3 \mathrm{msec}$ pulse width) since excitation would occur in both polarities of the stimulating electrode (Fuller, 1975). In this study, neurons behaving like the one in Figure $1 C$ were considered to be nonresponsive to LHA stimulation. In some experiments, the contralateral LHA and the ipsi- lateral hypothalamic ventromedial nucleus were electrically stimulated; stimulation of these sites did not induce slow potentials as evoked by the ipsilateral LHA stimulation.

All of the neuron discharges elicited by electrical stimulation of the tongue were also tested with taste, mechanical, and thermal stimulation of tongue receptors. Although some single gustatory neurons in the NTS responded to the other tongue modalities (mechanical or thermal) as mentioned earlier (Makous et al., 1963; Blomquist and Antem, 1965), they usually responded vigorously to only one of the stimulus modalities, and thus all of the neurons could be arbitrarily classified into three groups: gustatory, mechanical, and thermal neurons. Of a total of 37 neurons, gustatory, mechanical, and thermal neurons consisted of 17 (5), 14 (5), and 6 (4) units, respectively; the neurons in parentheses were nonresponsive to the LHA stimulation.

The latency of spike discharge induced by tongue and/ or the LHA stimulation was measured in these three types of neurons and is shown in Figure 2. The latency for each neuron was obtained by averaging the values of 10 trials. Gustatory, mechanical, and thermal neurons are indicated by solid, hatched, and open columns, respectively. The upper histogram represents the latencies of response to tongue stimulation. The latencies to tongue stimulation ranged from 3 to $25 \mathrm{msec}$, and their distribution was unimodal with a mean latency of $8.7 \pm$ $5.1 \mathrm{msec}$ (mean $\pm \mathrm{SD}, n=37$ ). Contrary to this, as shown in the lower histogram, the latencies to the LHA stimulation were smoothly scattered with a range of 3 to 44 msec and a mean latency of $20.7 \pm 12.7 \mathrm{msec}$ (mean \pm $\mathrm{SD}, n=23$ ). This wide range of distribution probably reflects multisynaptic connections between the LHA and the NTS neurons as compared with the simple synaptic connection between the tongue afferent fibers and the NTS neurons. In both cases, there were not statistically significant differences among mean latencies separately obtained in gustatory, mechanical, and/or thermal neurons ( $p>0.1$, the Student's $t$ test).

The modulatory effects from LHA activity upon the NTS neurons were analyzed by applying the conditioning stimulation of the LHA prior to the test electrical stim-

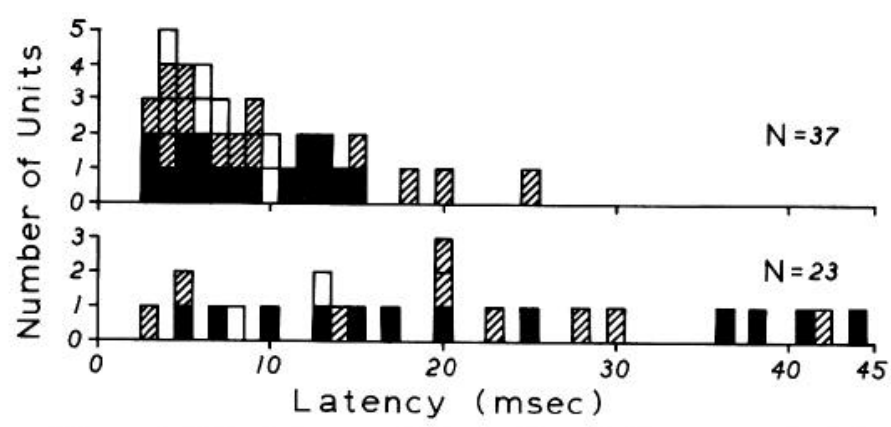

Figure 2. The latency of the spike discharges evoked by electrical tongue stimulation (upper histogram) in 37 NTS neurons and by the LHA stimulation (lower histogram) in 23 NTS neurons. The latency for each neuron was measured by averaging the values of 10 trials. Solid columns, gustatory neurons; hatched columns, mechanical neurons; open columns, thermal neurons. 
ulation of the tongue. The traces in Figure 3 show the influence of a conditioning stimulus which was recorded in gustatory (Fig. 3A1) and in mechanical neurons (Fig. (3A2). A typical facilitatory effect of the conditioning stimulus was observed in this gustatory neuron at a conditioning-test interval of $20 \mathrm{msec}$, i.e., an increase in the number of spikes per test stimulus and a slight reduction in spike latency (about $1 \mathrm{msec}$ ). On the other hand, a suppressive effect was evident in the mechanical neuron at a conditioning-test interval of $30 \mathrm{msec}$, as indicated by an absence of production of spikes in response to the test stimulus. The continuous traces of Figure 3, B1 and B2 demonstrate that the gustatory (Fig. $3 A 1)$ and the mechanical (Fig. 3A2) neurons responded to $0.01 \mathrm{M} \mathrm{HCl}$ solution and stroking the tongue surface with a glass rod, respectively. This series of experiments was performed in the neurons which were dually responsive to tongue and to LHA stimulation. In the neurons which were not responsive to LHA stimulation, the conditioning effect was not evident except for one case of a mechanical neuron which showed a clear suppressive effect at conditioning-test intervals of about 15 to 150 msec.

The results obtained at varying conditioning-test intervals are shown graphically in Figures 4 and 5 , in which the effect of conditioning stimulation was expressed as a percentage when the number of spikes induced by the test stimulus was divided by that induced by the control tongue stimulus. Figure 4 shows the results obtained from 12 gustatory neurons. Although the time course and the magnitude of the effects varied in different preparations, the conditioned responses of eight neurons were facilitated by 30 to $80 \%$ of their control level at a period of approximately 20 to $150 \mathrm{msec}$. The conditioned response of the remaining four neurons was not noticeably different from the unconditioned response. Since the effects of the LHA stimulation were variable and the gustatory neurons studied here tended to respond to more than one of the four basic taste qualities as previously reported (Makous et al., 1963; Doetsch and Erickson, 1970), relationships between the pattern of the effects of LHA stimulation and the differential sensitivity to the various chemical stimuli of the tongue were not found.

On the other hand, the conditioning effects observed in 14 mechanical and 2 thermal (indicated by broken line) neurons were, on the whole, suppressive, as illustrated in Figure 5. The time course and the magnitude of the effects were also variable among the neurons, but in 12 mechanical and 2 thermal neurons a clear suppressive effect lasting 50 to $300 \mathrm{msec}$ was observed. One of
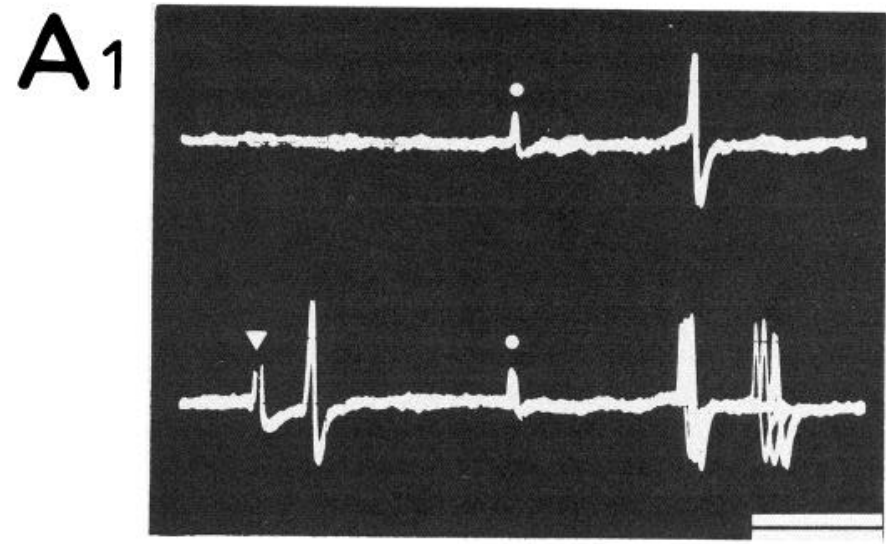
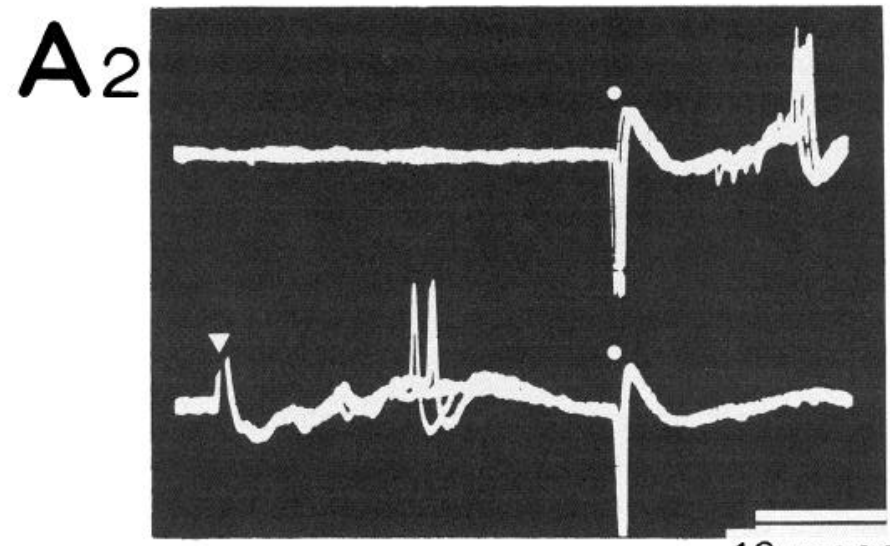

$10 \mathrm{msec}$
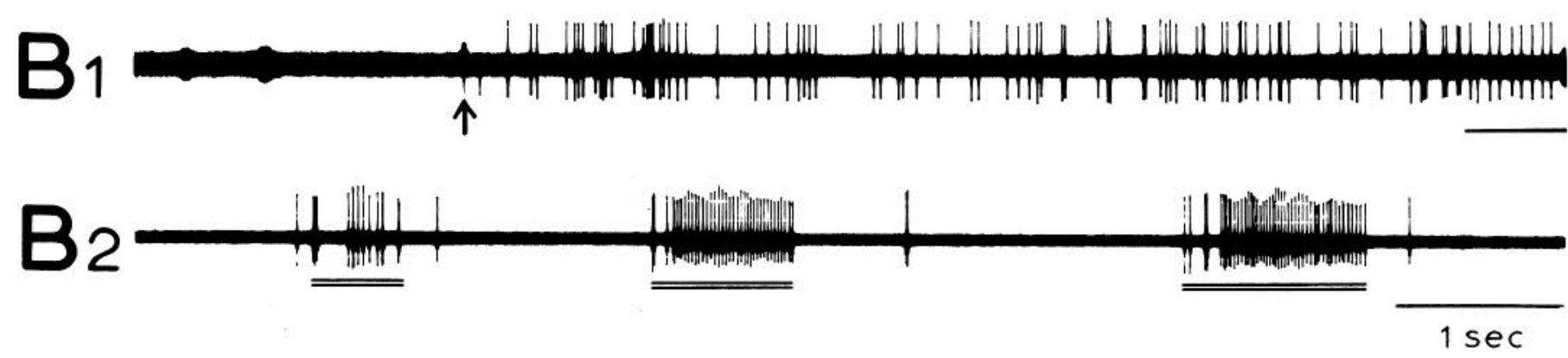

Figure 3. Facilitatory and suppressive effects of conditioning stimulation of the LHA. A1, Spike discharges by test stimulus of the tongue are shown in the upper record. A conditioning stimulus of the LHA was applied 20 msec prior to the test stimulus in the lower record. Circles and triangles indicate test and conditioning stimulations, respectively. Each record is five superimposed traces. Facilitation is shown by a decrease in latency of the first spike and by an increase in the number of spikes. A2, Response to test stimulus is in the upper record. A conditioning stimulus applied $30 \mathrm{msec}$ prior to the test stimulus suppressed spike discharges produced by the test stimulus, as seen in the lower record. B1, The neuron in $A 1$ responded to a $0.01 \mathrm{M} \mathrm{HCl}$ solution applied to the tongue at the arrow. B2, The neuron in $A 2$ responded to pressure of the tongue surface indicated by the double bars. Time calibrations indicated by bars are $10 \mathrm{msec}$ for $A 1$ and $A 2$ and 1 sec for $B 1$ and $B 2$. 


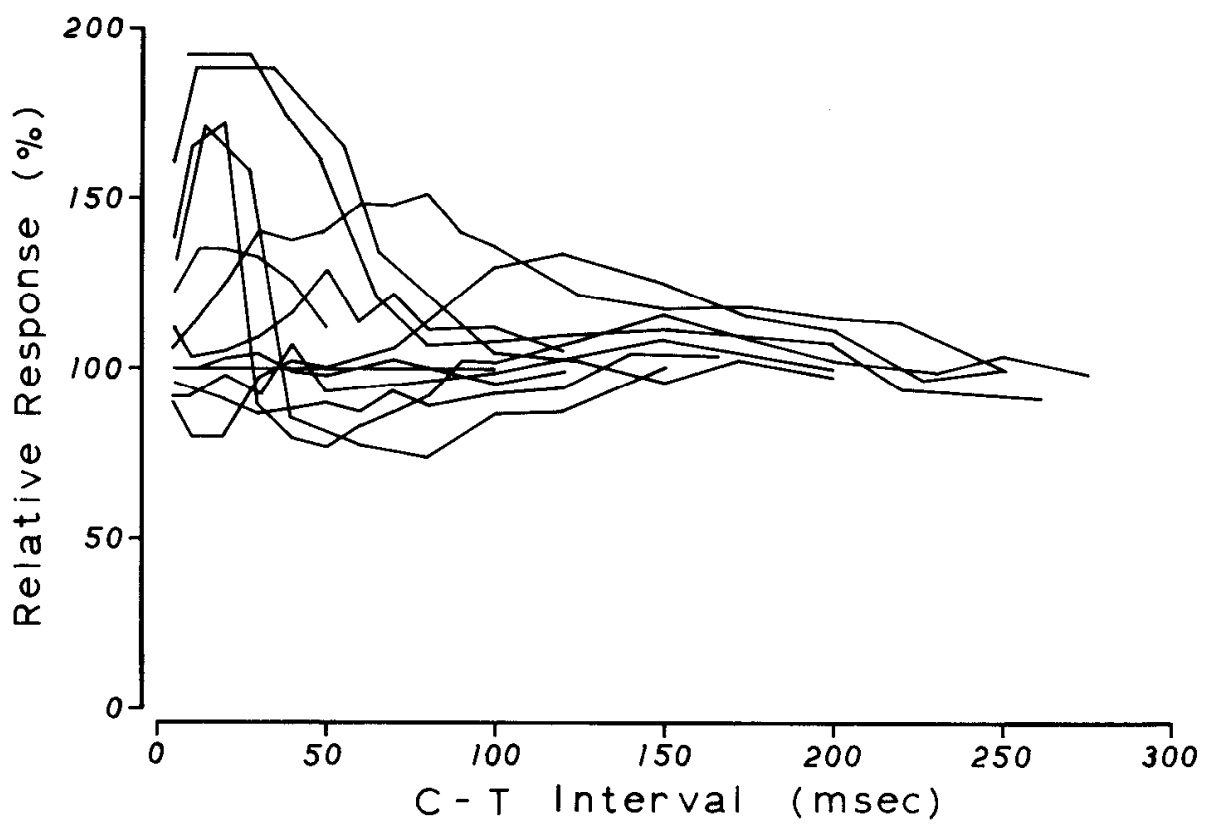

Figure 4. Time course of effect of conditioning LHA stimulation upon gustatory neurons. The relative response to test stimulus of the tongue is expressed as a percentage on the ordinate; each magnitude was obtained by averaging the value of 10 trails, which was calculated by dividing the number of impulses per test stimulus by that per control tongue stimulus. Of 12 neurons, 8 were facilitated by 30 to $80 \%$ of their control level for approximately a 20 - to $150-\mathrm{msec}$ period. The remaining 4 were unaffected.

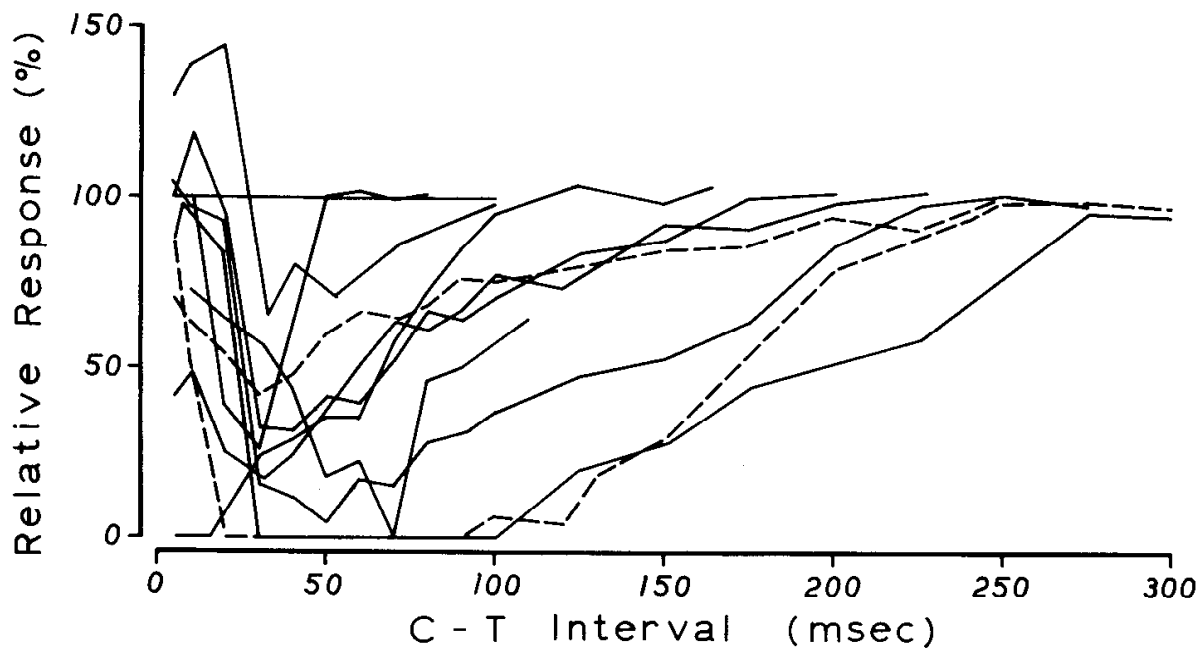

Figure 5. Time course of effect of conditioning LHA stimulation upon mechanical and thermal neurons. A suppressive effect lasting 50 to $300 \mathrm{msec}$ is seen in 12 mechanical (solid line) and 2 thermal (broken line) neurons. The other 2 mechanical neurons showed either no effect and/or an initial facilitatory effect followed by a suppression lasting about $150 \mathrm{msec}$.

the remaining two mechanical neurons showed a brief facilitatory effect ( 10 to $30 \mathrm{msec}$ ) followed by a suppression lasting about $100 \mathrm{msec}$; the other showed no effect.

\section{Discussion}

A major finding in the present study is that $62 \%$ of the NTS neurons responsive to gustatory, mechanical, and/or thermal stimulation of the anterior part of the tongue were also responsive to electrical stimulation applied to the ipsilateral LHA at the level of the ventro- medial nucleus. These NTS neurons variably responded to the LHA stimulation; that is, single or sometimes multiple spike discharges followed stimulation at frequency less than $20 \mathrm{~Hz}$; these showed an unstable latency ranging from 3 to $44 \mathrm{msec}$. In contrast, electrical stimulation of the tongue (up to 30 to $50 \mathrm{~Hz}$ ) produced single spike discharges with little variation of latency; the mean latencies ranged from 3 to $25 \mathrm{msec}$. These response characteristics indicate that the synaptic connection between the LHA neurons and the NTS neurons is poly- 
synaptic and more complicated than a simple synaptic connection as seen between the first-order tongue afferent and the second-order NTS neurons. Neuroanatomical studies revealed that the LHA neurons projected directly to the NTS as well as to nuclei in the lower brainstem, such as the parabrachial nucleus, the spinal trigeminal nucleus, and the locus ceruleus (Saper et al., 1976; Cedarbaum and Aghajanian, 1978; Roberts, 1980; Hosoya and Matsushita, 1981). Interestingly, physiological evidence has been obtained that the parabrachial nucleus is a gustatory relay area (Norgren and Leonard, 1973) and that the spinal trigeminal nucleus and the locus ceruleus are related to sensory stimuli of the oral region (Darian-Smith, 1966; Korf et al., 1974). These relay areas for the sensory afferent from the tongue are possibly involved in the descending pathways from the LHA neurons.

The LHA was demonstrated by both neuroanatomical and electrophysiological studies in rats to receive gustatory afferents (Norgren, 1970, 1976). This fact and present data indicate that the NTS neurons are submitted to a centrifugal control from the LHA which is one of the loci of gustatory inputs. Similar centrifugal controls within the gustatory system were demonstrated between cortical and thalamic taste cells (Yamamoto et al., 1980) and between the parabrachial taste area and the central amygdaloid nucleus which received gustatory afferents (Norgren, 1976). In addition, a feedback loop in another oral sensory system was reported between locus ceruleus and cells in the spinal trigeminal nucleus responding to tooth pulp stimulation (Igarashi et al., 1979).

When the conditioning stimulation of the LHA was applied prior to the test stimulation of the tongue, different modulatory effects were observed among the gustatory, mechanical, and thermal NTS neurons. Namely, the activity of the gustatory neurons tended to be facilitated by 30 to $80 \%$ of their control level for an approximately 20 - to $150-\mathrm{msec}$ period, while the activity of the mechanical and thermal neurons tended to be suppressed for a 50- to 300-msec period after the LHA stimulation. Since these conditioning effects were relatively longlasting, facilitatory or suppressive effects may be summated when the LHA neurons discharge at high frequency. According to experiments in freely moving rats (Hamburg, 1971; Ono et al., 1981), some neurons in the LHA were related to feeding acts, which showed spontaneous firing rates of less than $10 \mathrm{~Hz}$. They decreased or completely stopped producing spike discharges during eating food or licking taste solutions. Therefore, there is a possibility that before eating behavior the gustatory NTS neurons increase their reactivity to the gustatory inputs by the LHA activity and that during eating their reactivity is not modified. As to the mechanical and thermal NTS neurons, their reactivity may be suppressed as a result of LHA neuron discharge at more than $4 \mathrm{~Hz}$ before eating behavior.

As the NTS neurons responsive only to tongue stimulation were investigated in this study, the interpretation of modulatory effects from the LHA on the NTS neurons depends on an understanding of the functional significance of afferents from the tongue. It is known that sensory stimulation of the tongue, especially gustatory stimulation, reflexly induces salivary secretion as well as movement of the tongue and jaw in decerebrate rats (Emmelin, 1967; Yamamoto et al., 1982). In addition, there are some reports suggesting that these NTS neurons might be involved in gustatory-related endocrine gland activities (see Louis-Sylvestre and Le Magnen, 1980). The present study suggests that these gustatoryrelated endocrine activities and autonomic reflexes are possibly modulated by the LHA activity.

\section{References}

Bereiter, D., H. R. Berthoud, and B. Jeanrenaud (1980) Hypothalamic input to brain stem neurons responsive to oropharyngeal stimulation. Exp. Brain Res. 39: 33-39.

Blomquist, A. J., and A. Antem (1965) Localization of the terminals of the tongue afferents in the nucleus of the solitary tract. J. Comp. Neurol. 124: 127-130.

Cedarbaum, J. M., and G. K. Aghajanian (1978) Afferent projections to the rat locus coeruleus as determined by a retrograde tracing technique. J. Comp. Neurol. 178: 1-16.

Darian-Smith, I. (1966) Neural mechanisms of facial sensation. Int. Rev. Neurobiol. 9: 301-395.

Doetsch, G. S., and R. P. Erickson (1970) Synaptic processing of taste-quality information in the nucleus tractus solitarius of the rat. J. Neurophysiol. 33: 490-507.

Emmelin, N. (1967) Nervous control of salivary glands. In Handbook of Physiology. Sect. 6: Alimentary Canal II, pp. 595-632, American Physiological Society, Washington, D. C.

Fuller, J. H. (1975) Brain stem reticular units: Some properties of the course and origin of the ascending trajectory. Brain Res. 83: 349-367.

Halpern, B. P., and L. M. Nelson (1965) Bulbar gustatory responses to anterior and to posterior tongue stimulation in the rat. Am. J. Physiol. 20.9: 105-110.

Hamburg, M. D. (1971) Hypothalamic unit activity and eating behavior. Am. J. Physiol. 220: 980-985.

Hosoya, Y., and M. Matsushita (1981) Brainstem projections from the lateral hypothalamic area in the rat, as studied with autoradiography. Neurosci. Lett. 24: 111-116.

Igarashi, S., M. Sasa, and S. Takaori (1979) Feedback loop between locus coeruleus and spinal trigeminal nucleus neurons responding to tooth pulp stimulation in the rat. Brain Res. Bull. 4: 75-83.

Korf, J., B. S. Bunney, and G. K. Aghajanian (1974) Noradrenergic neurons: Morphine inhibition of spontaneous activity. Eur. J. Pharmacol. 25: 165-169.

Le Magnen, J. (1983) Body energy balance and food intake: A neuroendocrine regulatory mechanism. Physiol. Rev. 63: 314-386.

Louis-Sylvestre, J., and J. Le Magnen (1980) Palatability and preabsorptive insulin release. Neurosci. Behav. Rev. 4 (Suppl. 1): $43-46$.

Makous, W., S. Nord, B. Oakley, and C. Pfaffmann (1963) The gustatory relay in the medulla. In Olfaction and Taste, $\mathrm{Y}$. Zotterman, ed., pp. 381-393, Pergamon Press, New York.

Marshall, J. F., B. H. Turner, and P. Teitelbaum (1971) Sensory neglect produced by lateral hypothalamic damage. Science 174: $523-525$.

Norgren, R. (1970) Gustatory responses in the hypothalamus. Brain Res. 21: 63-77.

Norgren, R. (1976) Taste pathways to hypothalamus and amygdala. J. Comp. Neurol. 166: 17-30.

Norgren, R., and C. M. Leonard (1973) Ascending central gustatory pathways. J. Comp. Neurol. 150: 217-238.

Ono, 'T., H. Nishino, K. Sasaki, M. Fukuda, and K. Muramoto (1981) Long-term lateral hypothalamic single unit analysis 
and feeding behavior in freely moving rats. Neurosci. Lett. 21: $99-104$.

Powley, T. L. (1977) The ventromedial hypothalamic syndrome, satiety, and a cephalic phase hypothesis. Psychol. Rev. 84: 89-126.

Riley, J. N., J. P. Card, and R. Y. Moore (1981) A retinal projection of the lateral hypothalamus in the rat. Cell Tissue Res. 214: 257-269.

Roberts, W. W. (1980) $\left[{ }^{14} \mathrm{C}\right]$ Deoxyglucose mapping of firstorder projections activated by stimulation of lateral hypothalamic sites eliciting gnawing, eating, and drinking in rats. J. Comp. Neurol. 194: 617-638.

Rolls, E. T., M. J. Burton, and F. Mora (1976) Hypothalamic neuronal responses associated with the sight of food. Brain
Res. 111: 53-66.

Saper, C. B., A. D. Loewy, L. W. Swanson, and W. M. Cowan (1976) Direct hypothalamo-autonomic connections. Brain Res. 117: 305-312.

Scott, J. W., and C. Pfaffmann (1972) Characteristics of responses of lateral hypothalamic neurons to stimulation of the olfactory system. Brain Res. 48: 251-264.

Yamamoto, T., R. Matsuo, and Y. Kawamura (1980) Corticofugal effects on the activity of thalamic taste cells. Brain Res. 193: 258-262.

Yamamoto, T., T. Fujiwara, R. Matsuo, and Y. Kawamura (1982) Hypoglossal motor nerve activity elicited by taste and thermal stimuli applied to the tongue in rats. Brain Res. 238: 89-104. 